\title{
Observation of negative differential resistance in DNA molecular junctions
}

\author{
Ning Kang, ${ }^{\text {a) }}$ Artur Erbe, ${ }^{\text {b) }}$ and Elke Scheer \\ Fachbereich Physik, Universität Konstanz, D-78457 Konstänz, Germany
}

(Received 1 September 2009; accepted 18 December 2009; published online 12 January 2010)

\begin{abstract}
The mechanically controllable break junction technique is used to study charge transport through suspended DNA molecules. The current-voltage $(I-V)$ characteristics in an aqueous solution display series of negative differential resistance (NDR) and hysteresis behavior. Under high-vacuum conditions, the peak positions of NDR shift to lower voltage, and the amplitude is reduced dramatically. The observed NDR behavior is consistent with the polarization mechanism in DNA molecular junctions, which demonstrates a change in the coupling of the molecular level to a polaron mode under different environment.
\end{abstract}

Nonlinear charge transport in molecules is a central issue of research in the development of molecular electronics. ${ }^{1,2}$ Recently, the characteristic decreasing current with increasing voltage in particular voltage ranges has been observed in molecular ensembles and referred to as negative differential resistance (NDR) or NDR-like features. ${ }^{2,3}$ It is of special interest because of its potential application in the realization of logic devices and memory circuits. In view of the device application, the exhibiting NDR must be robust and reproducible with the large peak-to-valley ratio. Therefore, the fundamental understanding of the mechanisms of NDR or NDR-like behavior in molecular junctions is a key issue for the future developments. A main challenge remains the realization of controllable NDR devices by virtue of internal degrees of freedom of the molecules, and in particular in single-molecule devices. Recently, there has been a growing interest in the polarization response, i.e., combined effects of charging and conformational change, which provides an important route to understand and design NDR and switching behavior in the molecular junctions. ${ }^{4-7}$ Molecular electronics based on DNA molecules provide an ideal platform to investigate polaron transport in molecular junction. DNA is a dynamic structure, in which charge transfer might be related to the molecular vibrations and affected by conformational change.

In this letter, we report room temperature charge transport measurements performed on DNA molecules by use of the mechanically controllable break-junction (MCBJ) technique. Multiple NDR-like peaks are observed through molecular junctions with hysteresis behavior at high bias voltage. The peak positions exhibit shifts to lower voltage under vacuum conditions. These observations are consistent with a polaron mechanism including the effects of charging and polarization.

Previously, we had developed a protocol using the MCBJ technique contacting single clusters or molecules. ${ }^{8,9}$ This technique provides a stable device structure, which allows to controllably adjust the separation between junctions and to measure the charge transport of the device simulta-

\footnotetext{
a) Present address: Laboratoire de Physique des Solides, Université ParisSud, 91405 Orsay, France. Electronic mail: kang@lps.u-psud.fr.

${ }^{b}$ Present address: Forschungszentrum Dresden-Rossendorf, D-01328 Rossendorf, Germany
}

neously. The sample is mounted in a three-point mechanical bending apparatus. By bending the substrate, the metallic bridge can be broken, creating two atomic-sized metal electrodes, which are of right size to contact single molecules. We use a liquid cell which consisted of a tube that was gently pressed against the substrate from above. We create molecular junctions by repeatedly breaking and linking the junction in TE buffer solution ( $10 \mathrm{mM}$ Tris- $\mathrm{HCl}, \mathrm{pH} 8.0$, and $1 \mathrm{mM}$ EDTA) containing $25 \mu \mathrm{M}$ DNA molecules. Upon applying a bias voltage of $80-300 \mathrm{mV}$, charged molecules will be attracted to bridge the gap between freshly broken $\mathrm{Au}$ electrodes and can thus form a molecular junction [Fig. 1(a)].

In our experiments, we use double-stranded $10 \mathrm{~nm}$ long DNA oligomers with the following sequence: 5 ' thiol-dGGGC GGC GAC CTT CCC GCA GCT GGT ACG GAC. The molecules are functionalized with thiol groups at both ends, which bind the molecules to the gold surfaces. ${ }^{9,10}$ It is noteworthy that the persistence length of DNA in aqueous solution is $40-50 \mathrm{~nm}$, which is much larger than the length of molecules in this experiment so that DNA molecules are suspended above the substrate and clamped at both ends by linking groups as linear and stiff wires. The advantage of preparing a free-standing molecular junction is not only avoiding the effect of surface field ${ }^{11}$ but also being more suitable for exploring the interplay between charge transport and mechanical motion in the device. (a)

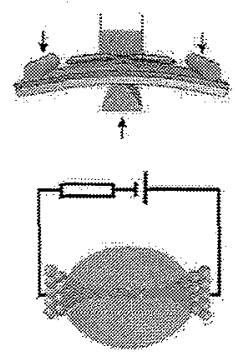

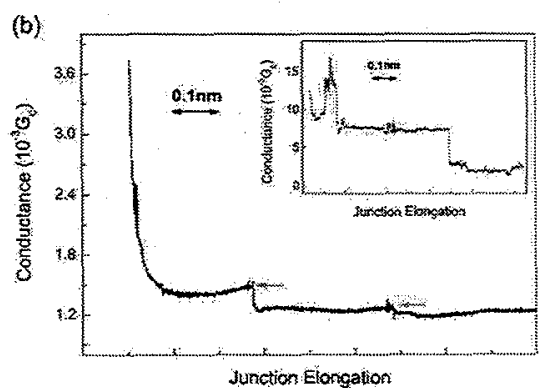

FIG. 1. (Color online) (a) Schematic drawing of the MCBJ setup and electrical circuit used to trap the DNA molecules. (b) Representative conductance trace measured while breaking gold bridges in DNA solution with a stretching rate of $\sim 150 \mathrm{pm} / \mathrm{s}$. The trace exhibits step-wise changes at conductance values below $G_{0}$ corresponding to conduction through a varying number of DNA molecules bridging across the gold tips. The traces vary from device to device. The inset displays another sample trace recorded at different stretching rate $(\sim 80 \mathrm{pm} / \mathrm{s})$. 

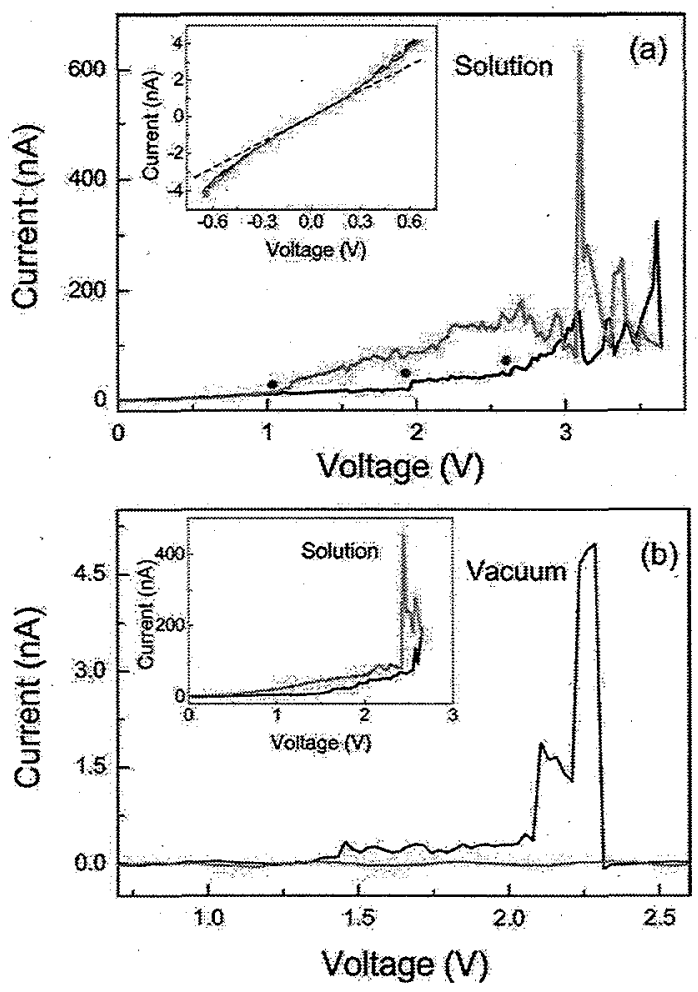

FIG. 2. (Color online) Typical $I-V$ characteristic of DNA molecular junction showing NDR peaks. The black curves are the forward sweep, and the red curves are the backward sweep with a sweep rate of $50 \mathrm{mV} / \mathrm{s}$. (a) The measurement was performed in aqueous solution. At positive bias, multiple NDR events are observed and manifested by hysteretic behavior. The current exhibits steplike jumps in a bias range between 1 and $3 \mathrm{~V}$ (indicated with blue dots). Inset is a magnified $I-V$ characteristic at low bias showing a nonlinear behavior above $0.2 \mathrm{~V}$. (b) The measurement taken for the same junction in the vacuum exhibits similar NDR events. Note that the magnitude of the NDR peaks is reduced and the voltage values of the NDR peaks shift to lower bias. The inset shows NDR peaks from a different device in the solution.

Comparing our previous trapping process reported in Ref. 9, we found that a liquid environment offers better control on the contacting of DNA molecules within junction. While fresh Au contacts are pulled and broken in the solution, the discrete conductance plateaus were observed at values below $G_{0}$, the quantum of conductance, that relate to conduction through a small number of molecules bridging the $\mathrm{Au}$ point contacts [see Fig. 1(b)]. A contrastive experiment done in solution without molecules showed no such low-conductance step.

When the junction was stretched until the lowest conductance plateau, being attributed to the formation of a single DNA junction, we stopped the motor and recorded the current while sweeping the bias voltage in the solution. Figure $2(a)$ shows a representative current-voltage $(I-V)$ characteristic of DNA molecular junctions. Starting from $0 \mathrm{~V}$, the molecules are in the low conductance state with nonlinear behavior above $0.2 \mathrm{~V}$, being in agreement with previous reports $^{9,12,13}$ [see Fig. 2(a), inset]. The current initially remains less than $10 \mathrm{nA}$ and then gradually increases when the voltage exceeds $\sim 1 \mathrm{~V}$. Upon approaching $3.09 \mathrm{~V}$, the current drops abruptly to $60 \mathrm{nA}$, resulting in a first NDR peak with a peak-to-valley ratio of $3: 1$. As voltage increases further the consecutive NDR peaks appear at 3.27, 3.40, and $3.61 \mathrm{~V}$. It should be noted that for a given junction the NDR peaks were observed only in one bias direction, here the positive one. In other examples the peaks appeared for negative bias, strongly suggesting that individual or very few molecules contribute to the current. Assuming that the NDR effect is not a collective effect of many molecules one would expect the NDR effect to appear in both polarities due to ensemble averaging. A backward voltage sweep also exhibits dramatic NDR peaks with larger peak-to-valley ratios and a shift of the NDR peak positions. In addition, a hysteresis at high voltage is always present. The current returns to the low conductance state only when the applied bias falls below $1.10 \mathrm{~V}$.

We proceeded to conduct further $I-V$ measurements on the same junction in vacuum. The device is dried in a flow of nitrogen. Then the whole setup is mounted in an electromagnetically shielded box, which is pumped to a moderately high vacuum $\left(<10^{-7} \mathrm{mbar}\right)$. The volatile buffer can be completely removed in high vacuum. After pumping, the resistance is always very high $R>10^{11} \Omega$ if no molecules were solved in the solution. The $I-V$ measurements in the vacuum, seen in Fig. 2(b), show similar NDR events. A pronounced difference is observed when we compare the measured $I-V$ curve in vacuum with that in aqueous solution; the magnitude of the current in vacuum is much smaller than that in aqueous solution, and the voltage at which the NDR event occurs is reduced from the $-3.0 \mathrm{~V}$ to lower bias $\sim 2.0 \mathrm{~V}$. Approximately $10 \%$ of 60 break junctions exhibit a NDRlike feature with hysteresis, and $20 \%$ of devices display a nonlinear $I-V$ at high bias as reported previously. ${ }^{9}$ The data set included six junctions where the $I-V$ curves were recorded in both the solution and vacuum condition. Although the ratios of peaks-to-valleys varied from device to device, which may result from slightly varying molecules-electrode contact geometry, the positions of NDR peaks for all the junctions occur in a similar voltage range of $2-3.6 \mathrm{~V}$ [see inset of Fig. 2(b)]. The resistance of junctions in the vacuum was found to be about two orders of magnitude smaller than the same device in aqueous solution.

A consistent picture for multiple NDR peaks with hysteresis is a polaron-mediated charge transport through the molecular junction. ${ }^{4-6}$ The polaron is formed when the charge tunnels into the molecule and becomes trapped. The energy of resonant molecular level shifts by polaron formation that depends on the electronic population of the level, $n_{0}$ at energy $\varepsilon_{0} 5$

$$
\overline{\varepsilon_{0}}\left(n_{0}\right)=\varepsilon_{0}-2 \varepsilon_{\text {reorg }} n_{0} .
$$

As soon as the level population is high enough, the energy level is shifted by formation of a polaron. When the level energy moves outside the voltage bias window, the current is blocked as depicted in Fig. 3.

The structural flexibility of the DNA molecule has a crucial impact on the creation of a polaron responding to the injection of charge. Previous experimental and theoretical investigations have suggested that strong charge-lattice coupling and polaron formation contribute to the charge migration and transport in DNA. ${ }^{14,15}$ It is interesting to note that a subset of the $I-V$ characteristics shows steplike features in the range 1.0-3.0 V [blue dots, Fig. 2(a)], which give a signature of voltage-driven structural change or nonequilibrium polaron transport through DNA. ${ }^{16}$ 
(a) Initial state

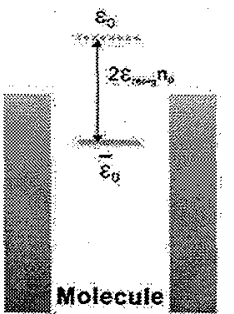

(b) Discharging

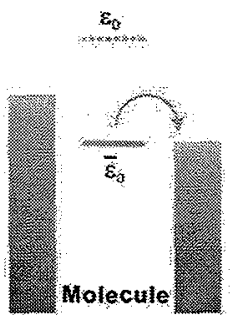

(c) NDR event

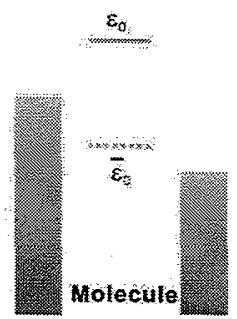

FIG. 3. (Color online) The observed NDR behavior in the molecular junction can be explained by a polaron model, which leads to a shift of molecular level upon charging. (a) The molecular level is initially charged with the molecular level $\varepsilon_{0}$ being located above the Fermi energy of the electrodes When a polaron forms the localized charge within the molecule occupies the polaron level $\overline{\varepsilon_{0}}$ within the bias window resulting in a current increase. (b) Further increase in the bias voltage causes discharging of the polaron level. When the electrode's Fermi level aligns with the polaron level, the current through the junction reaches a peak of NDR. (c) When the charge leaves the molecule, the resonant level moves away and no molecular level lies in the bias window, resulting in a pronounced drop of the current through the molecule.

The observed NDR behavior in the DNA junctions can be understood beginning in the filled level situation. At low bias, the molecule initially is in the charged state as shown in Fig. 3(a). When the voltage is increased, the occupied molecular level approaches resonance with the Fermi levels of the leads, and charge tunnels out the molecule. The $I-V$ curve then exhibits the discharging process in a characteristic manner. Increasing the bias voltage gives rise to a drop of current through the junction. The Fermi level will approach the next molecular levels with further increase in the bias voltage. Therefore multiple NDR events may appear, as shown in Fig. 3 . It is important to emphasize that the shape of the NDR peak in a backward sweep is similar to forward case due to charging or discharging of the molecular level. The hysteretic $I-V$ characteristics can also be understood within the polaron model, where the self-consistency condition results in multiple stable solutions for $n_{0}{ }^{4}$

When the junction is introduced into vacuum conditions, the mechanical strength of the DNA molecules and electronic coupling to the vibronic modes would be dramatically modified. Due to the polarization of the water and the ions, the solution has also a strong effect in further driving and stabilizing the polaron formation within the molecule. ${ }^{15}$ As mentioned above, NDR occurs at the bias voltage when the charge leaves the molecule matching the resonant condition, i.e., $\mu \approx \varepsilon_{0}-2 \varepsilon_{\text {reorg }} n_{0}$. The polaron reorganization energy, $\varepsilon_{\text {reorg }}$, is reduced with weaker vibronic coupling strength in the vacuum, and the polaron level is lifted with respect to the Fermi level of the leads. Thus, the decrease in $\varepsilon_{\text {reorg }}$ results in a shift of the NDR peak to lower voltage in the vacuum.
Other proposed explanations for the NDR behavior involve conformational change or local heating induced by voltage. ${ }^{17,18}$ Recent experiments have shown a nonmonotonic behavior of the effective local temperature of the molecular junction under high biases. ${ }^{18}$ For an analytical description of the observed NDR peaks in DNA junctions, an extended theory would be required that involves a different voltage dependence of local heating in the solvent and vacuum environment.

In conclusion, we have studied charge transport in freestanding DNA molecules bridged across atomic-size gold contacts using the MCBJ technique. The current-voltage characteristics reveal multiple NDR peaks and hysteresis behavior, which is consistent with a polaron assisted charge transfer mechanism in molecular junction. The decrease in polaron coupling in vacuum leads to a reduction in amplitude of the NDR peaks and a shift of the resonant level position to lower bias voltage. For DNA-based electronics applications, these results can provide guidance in designing and employing DNA molecules as active circuit elements.

We thank A. Marx, M. Hettler, G. Cuniberti, and M. Elstner for fruitful discussions and T. Böhler for his contributions to the experiment. We acknowledge financial support by the Deutsche Forschungsgemeinschaft under Grant Nos. SFB 513 and SPP 1243, the Krupp Foundation (Alfried Krupp Prize), and the Landesstiftung Baden-Württemberg (Kompetenznetz Funktionelle Nanostrukturen).

A. Nitzan and M. A. Ratner, Science 300, 1384 (2003).

${ }^{2}$ N. J. Tao, Nat. Nanotechnol. 1, 173 (2006).

${ }^{3}$ J. Chen, M. A. Reed, A. M. Rawlett, and J. M. Tour, Science 286, 1550 (1999).

${ }^{4}$ M. Galperin, M. A. Ratner, and A. Nitzan, Nano Lett. 5, 125 (2005).

${ }^{5}$ M. Galperin, M. A. Ratner, A. Nitzan, and A. Troisi, Science 319, 1056 (2008).

${ }^{6}$ S. Yeganeh, M. Galperin, and M. A. Ratner, J. Am. Chem. Soc. 129, 13313 (2007).

${ }^{7}$ A. Mitra, I. Aleiner, and A. J. Millis, Phys. Rev. Lett. 94, 076404 (2005).

${ }^{8}$ T. Böhler, J. Grebing, A. Mayer-Gindner, H. v. Löhneysen, and E. Scheer, Nanotechnology 15, S465 (2004).

${ }^{9}$ N. Kang, A. Erbe, and E. Scheer, New J. Phys. 10, 023030 (2008).

${ }^{10}$ B. Bornemann, S. P. Liu, A. Erbe, E. Scheer, and A. Marx, ChemPhysChem 9, 1241 (2008).

${ }^{11}$ A. Y. Kasumov, D. V. Klinov, P. E. Roche, S. Guéron, and H. Bouchiat, Appl. Phys. Lett. 84, 1007 (2004).

${ }^{12}$ B. Q. Xu, P. M. Zhang, X. L. Li, and N. J. Tao, Nano Lett. 4, 1105 (2004).

${ }^{13}$ H. Cohen, C. Nogues, R. Naaman, and D. Porath, Proc. Natl. Acad. Sci. U.S.A. 102,11589 (2005).

${ }^{14}$ K. H. Yoo, D. H. Ha, J. O. Lee, J. W. Park, J. Kim, J. J. Kim, H. Y. Lee, T. Kawai, and H. Y. Choi, Phys. Rev. Lett. 87, 198102 (2001).

${ }^{15}$ E. M. Conwell, Proc. Natl. Acad. Sci. U.S.A. 102, 8795 (2005).

${ }^{16}$ B. B. Schmidt, M. H. Hettler, and G. Schön, Phys. Rev. B 77, 165337 (2008).

${ }^{17}$ B. Song, M. Elstner, and G. Cuniberti, Nano Lett. 8, 3217 (2008).

${ }^{18}$ Z. F. Huang, F. Chen, R. D'Agosta, P. A. Bennett, M. Di Ventra, and N. J. Tao, Nat. Nanotechnol. 2, 698 (2007). 